\title{
La arbitrabilidad subjetiva en el contrato de fiducia en Colombia
}

Subjective Arbitrability in Colombian Trust Contracts

A arbitrabilidade subjetiva no contrato de fidúcia na Colômbia

\section{Sebastián ORTEgón OBAndo*}

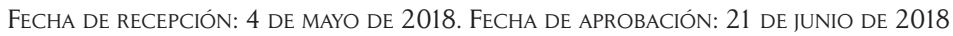

DoI: http://dx.doi.org/10.12804/revistas.urosario.edu.co/sociojuridicos/a.6780

Para citar este artículo: Ortegón Obando, S. (2019). La arbitrabilidad subjetiva en el contrato de fiducia en Colombia. Estudios Socio-Jurídicos, 21(1), 177-207. Doi: http://dx.doi.org/10.12804/revistas.urosario.edu.co/ sociojuridicos/a.6780

\section{RESUMEN}

El objetivo del presente artículo es hacer una reflexión práctica sobre los problemas alrededor de los pactos arbitrales en materia de contratos de fiducia. En particular, sobre los problemas que pueden surgir para los no signatarios del pacto arbitral. La metodología empleada para la elaboración del presente estudio fue el análisis dogmático de casos jurisdiccionales, así como de la doctrina nacional e internacional sobre la materia. En primer lugar, se revisan las diferentes aproximaciones teóricas que se han realizado con el fin de caracterizar lo que este artículo cataloga como dos tendencias en la materia: rígida y flexible. Posteriormente, se estudian algunas hipótesis prácticas que pueden surgir, dada la estructura del contrato, para demostrar que las figuras que en la actualidad son utilizadas pueden redundar en la violación de los derechos de terceros.

Palabras clave: fiducia, pacto arbitral, arbitrabilidad subjetiva, tercero no signatario.

* Abogado de la Universidad del Rosario. Estudiante de maestría de la Facultad de Jurisprudencia de la Universidad del Rosario. Profesor auxiliar de la Cátedra de Introducción al Derecho Civil de la misma Universidad. ORCID: https://orcid.org/0000-0001-5370-101X. Correo electrónico: sebastian.ortegon@urosario.edu.co 


\section{ABSTRACT}

The aim of this article is to analyze in a practical way, about the issues that arrives around the arbitration clauses related with trust contracts. Particularly, problems related with non-signatories are studied. The article is made based on a case study methodology, as well as on a national and international doctrine review. The following structure was used: first, two tendencies related to the scope of action of subjective arbitrability have been identified, these were called rigid and flexible. Then, different practical hypothesis related with trust contracts have been studied, in order to explain the difficulties that might arise, particularly for the rights of third parties.

Keywords: Trustee or fiduciary, trust, arbitral agreement, non-signatories, subjective arbitrability.

\section{RESUMO}

O objetivo do presente artigo é fazer uma reflexão prática sobre os problemas ao redor dos pactos arbitrais em matérias de contratos de fidúcia. Particularmente, sobre os problemas que podem surgir para os não signatários do pacto arbitral. A metodologia empregada para a elaboração do presente estudo foi a análise dogmática de casos jurisdicionais, assim como da doutrina nacional e internacional sobre a matéria.

Em primeiro lugar, revisam-se as diferentes aproximações teóricas que se têm realizado com o fim de caracterizar o que este artigo cataloga como duas tendências na matéria: rígida e flexível. Posteriormente se estudam algumas hipóteses práticas que podem surgir dada a estrutura do contrato, para demonstrar que as figuras que na atualidade são utilizadas podem redundar na violação dos direitos de terceiros.

Palavras-chave: fidúcia, pacto arbitral, arbitrabilidade subjetiva, terceiro não signatário. 
El propósito del presente artículo es analizar algunas de las hipótesis relacionadas con la vinculación de terceros, o no signatarios, en un proceso arbitral a la luz del contrato de fiducia mercantil con el fin de demostrar la necesidad de repensar la noción de arbitrabilidad subjetiva en el marco de este tipo de vehículos jurídicos.

La naturaleza misma del arbitraje, como contrato con efectos procesales ${ }^{1}$, supone dificultades respecto de contratos que abarcan múltiples extremos negociables, como es el caso del contrato de fiducia mercantil. Resulta problemático que si la justificación primordial de la jurisdicción de la que está revestido un árbitro es la voluntad de las partes², la decisión tomada en un laudo pueda afectar derechos de personas que no han sido parte del pacto arbitral.

Tales cuestionamientos ponen de presente que la aplicación de cláusulas arbitrales en el contrato de fiducia, vehículo jurídico de gran utilización en el tráfico jurídico contemporáneo ${ }^{3}$, puede afectar derechos como el debido proceso de aquellas personas que, sin ser parte en el contrato, están interesadas en la solución de los conflictos que de él se deriven. Por tanto, el éxito de la aplicación en este tipo de mecanismos radica en que, además de que sean rápidos, especializados y definitivos, tengan reglas claras en cuanto a quiénes están cobijados por el pacto arbitral. Infortunadamente, tal certeza no existe en el sistema jurídico colombiano.

Es importante determinar que el presente trabajo se referirá exclusivamente a los problemas de la arbitrabilidad subjetiva o a los de la com-

1 No es objeto del presente estudio hacer una revisión profunda sobre la naturaleza sustancial o procesal del pacto arbitral. No obstante, debemos advertir que asumir una u otra noción trae como consecuencia efectos diversos respecto de los requisitos y criterios necesarios para la extensión de los efectos a terceros. Para ver más acerca de la discusión en lo atinente al derecho colombiano sobre el asunto ver Ramírez Baquero (2017).

2 Así está consagrado en el ordenamiento colombiano por mandato constitucional. El Artículo 116 de la Carta Política establece: "Los particulares pueden ser investidos transitoriamente de la función de administrar justicia en la condición de jurados en las causas criminales, conciliadores o en la de árbitros habilitados por las partes para proferir fallos en derecho o en equidad, en los términos que determine la ley" (cursiva fuera del texto).

3 De acuerdo con el informe anual de Asofiduciarias del año 2013, el incremento del sector fiduciario en el año anterior fue de $24 \%$, siendo el inversionista institucional más representativo del sistema financiero con una participación del 34,9\% (Asofiduciarias, 2014). 
petencia ratio personae, excluyendo aquellos que puedan surgir respecto de la denominada arbitrabilidad objetiva o competencia ratio materiae. De la misma manera, el objeto de estudio se limita a la fiducia mercantil $y$, por consiguiente, se excluye el encargo fiduciario con el objetivo de contemplar solamente las situaciones en las cuales se constituye un patrimonio autónomo.

Uno de los presupuestos iniciales para abordar el tema de la arbitrabilidad subjetiva en el contrato de fiducia mercantil es el hecho de que la jurisprudencia nacional, tanto jurisdiccional como arbitral, es bastante limitada, así como la doctrina que ha abordado este tema. En ese sentido, se recurrirá en varias ocasiones a la experiencia comparada y a la internacional con el fin de analizar posibles soluciones. Si bien es claro que existen diferencias marcadas en cuanto al arbitraje nacional e internacional, para efectos del objeto de estudio del presente artículo serán utilizados de forma indistinta. De la misma manera, no serán tenidas en cuenta las diferencias jurídicas entre los contratos de fiducia y trust en la medida en que entendemos que estas no alteran las problemáticas que surgen derivadas de los pactos arbitrales.

Para desarrollar el problema propuesto, en primer lugar, se abordarán los alcances de la teoría de la arbitrabilidad subjetiva frente a las facultades del tribunal y su competencia. Posteriormente, se presentarán las diferentes hipótesis que pueden surgir respecto del contrato de fiducia a la luz de la arbitrabilidad subjetiva.

\section{El doble concepto de parte y tercero desde la perspectiva contractual y procesal}

Antes de dar inicio al estudio específico del problema que nos ocupa, debemos advertir la presencia de una dificultad terminológica y conceptual relacionada con qué se entiende por parte y por tercero y su aplicación específica al pacto arbitral. De hecho, se trata de conceptos vagos y ambiguos en tanto pueden tener diferentes significados, dependiendo del contexto en el que se encuentren, y su aplicación a determinados casos no es suficientemente clara (Mantilla Espinosa, Salcedo Castro \& Bernate Ochoa, 2018). Como ya lo mencionamos, el carácter contractual 
con efectos procesales implica consultar el significado de las palabras desde una perspectiva sustancial y procesal. Así mismo, al dilucidar este concepto, surge, naturalmente, el de tercero cuya definición se construye por oposición al de parte, siendo tercero todo aquel que no es parte (Tobar Ordoñez, 2017).

Desde el punto de vista procesal, es parte toda persona afectada de forma directa por una sentencia que se profiere. En esa medida, existe identidad entre la figura del demandante y del demandado y la de parte, desde una perspectiva procesal (Tobar Ordoñez, 2017). Al respecto, es necesario manifestar que se trata de una postura que ha sido modificada a nivel colombiano como consecuencia de la expedición de un nuevo estatuto procesal, la Ley 1564 de 2012, que cataloga a cualquier persona que pueda ser afectada por la sentencia como parte, incluso en una categoría específica denominada "otra parte" en la que incluye a todas aquellas personas que puedan verse patrimonialmente afectadas por una sentencia judicial.

Desde una perspectiva sustancial o contractual, parte puede ser entendida como cualquier persona que concurra a un contrato por medio de la manifestación de su voluntad al momento de la celebración o ejecución del contrato y que dicha manifestación traiga como efecto la adquisición de derechos $\mathrm{u}$ obligaciones al patrimonio de la persona que es parte. Si bien algunos autores sostienen que la manifestación de la voluntad es el criterio fundamental para determinar la condición de parte, se identifican en la actualidad casos en los que se pueden hacer manifestaciones de la voluntad sin ser parte, o incluso ser parte como consecuencia de ficciones legales como en el caso de los sucesores a título universal (Pizarro Wilson, 2010).

Ahora bien, existe coincidencia entre ambos conceptos a nivel procesal al momento de proferir sentencia en la que el juez o árbitro debe analizar la llamada legitimación en causa por activa y pasiva, que es el estudio de la identidad que debe haber entre las personas que actúan como partes procesales y los titulares del derecho jurídico-sustancial, es decir las partes contractuales.

No obstante, se trata de conceptos diferentes que deben ser diferenciados en tanto solamente se está ante un problema de arbitrabilidad subjetiva en aquellos casos en los que una persona no coincida con los 
requisitos para ser parte contractual, mientras que si no los cumple para ser parte procesal, se estará ante un caso de capacidad para ser parte y, por tanto, excluido del presente análisis.

\section{El efecto relativo de los contratos}

En virtud del efecto relativo de los contratos, la regla general es que un acuerdo de voluntades solamente genera efectos respecto de sus partes. Por consiguiente, cualquiera que no sea parte del contrato es un tercero respecto de quien el contrato no debe generar ningún efecto, salvo el denominado efecto absoluto que implica el reconocimiento del contrato como un hecho en el mundo jurídico (Vidal Olivares, 2010).

Como consecuencia de lo anterior, a nivel patrimonial un tercero debe ser neutro a los contratos que otros celebren, de manera que los mismos no deben traducirse ni en derechos ni en obligaciones a su cargo.

Independientemente del origen histórico o filosófico de este principio (Pizarro Wilson, 2004), debemos advertir que el mismo explica la razón por la cual, por regla general, no es posible vincular a un tercero en un contrato, a la cláusula arbitral contenida en dicho contrato, en tanto ello implicaría atribuirle efectos patrimoniales a alguien que no debería ser afectado por un negocio jurídico sobre el que no prestó su consentimiento. Como se verá más adelante, las excepciones al efecto relativo surgen cuando la extensión de los efectos del contrato a un tercero le trae exclusivamente beneficios y no obligaciones para sí.

En esa medida, la pregunta que subyace a nuestra investigación es: en las cláusulas arbitrales incorporadas a contratos de fiducia, ¿cuándo es posible hacer excepciones al principio del efecto relativo de los contratos?

\section{La teoría de la arbitrabilidad subjetiva}

El arbitraje como mecanismo de solución de controversias constituye una excepción a la regla general, que es el monopolio de la administración de justicia por parte del Estado. En ese orden ideas, no todas las materias pueden ser sometidas al arbitraje, ni todas las personas pueden 
solucionar las controversias a través de este mecanismo. Cuando la cuestión de arbitrabilidad responde a la materia que va a ser objeto del tribunal, se trata entonces de un problema de arbitrabilidad objetiva. Por el contrario, si la cuestión de la arbitrabilidad responde a los sujetos para quienes es obligatoria la decisión del tribunal, entonces se está ante un caso de arbitrabilidad subjetiva.

La discusión alrededor de la manera de vincular a personas que no han firmado el pacto arbitral puede tener diferentes nombres según las legislaciones de que se trate (Born, 2014). Por tanto, una situación que se cuestione por la competencia ratio personae podría ser interpretada como un problema de arbitrabilidad objetiva por cuanto se busca solucionar una controversia que un pacto no abarca. Lo anterior se agudiza en el caso en el que una controversia incluye la decisión sobre derechos de terceros. En esa situación la competencia del tribunal para conocer puede ser considerada como un problema de arbitrabilidad objetiva. Al respecto, Namén Vargas (2000) sostiene que puede ser entendida como una prohibición en cuanto se trata de una materia restringida para las partes, en la medida en que genera efectos frente a terceros o incluso puede generar efectos erga omnes.

Así y todo, la competencia ratio personae tiene su fundamento en el principio de relatividad de los negocios jurídicos según el cual, para que un contrato produzca efectos sobre una persona, esta tiene que haber consentido en ello (Pizarro Wilson, 2010). Como el arbitraje surge como consecuencia de la intención de las partes, su manifestación de voluntad, en principio, debería afectar solamente a aquellas personas que han hecho tal manifestación (Born, 2014). Por consiguiente, la competencia del tribunal arbitral estará limitada subjetivamente por las personas que expresen su voluntad en ese sentido ${ }^{4}$.

La naturaleza jurídica del pacto arbitral, en su origen, es de estirpe contractual y, por tanto, se configura como un negocio jurídico ${ }^{5}$ (Namén

4 A diferencia de la legislación anterior, el Artículo 3 de la Ley 1563 de 2012 no contempla una forma específica para el pacto arbitral, de manera que el requisito de expresar la manifestación de la voluntad de someter una controversia a arbitraje de forma escrita estará satisfecho con una serie de hipótesis que, en últimas, permiten concluir que el pacto no necesariamente debe constar por escrito, como lo es la afirmación en una demanda de la existencia del pacto, sumado al silencio de la otra parte.

5 Al respecto, es importante aclarar que existe una discusión frente a la naturaleza misma 
Vargas, 2000). Así mismo, las reglas generales de los contratos le son aplicables. Si se tiene en cuenta que la competencia del árbitro se produce exclusivamente como consecuencia del negocio jurídico celebrado por las partes, es apenas razonable que esa competencia esté limitada frente a las personas que así lo quisieron en lo que es la manifestación general del efecto relativo de los contratos. Por tanto, dada la naturaleza contractual del pacto arbitral, resulta aparentemente muy claro que el axioma res inter alias acta le sea aplicable a los procedimientos arbitrales (Sinisterra Páez, 2010). Sin embargo, el inconveniente surge ante la situación en la que una persona que no ha firmado el pacto arbitral en realidad sí ha consentido en arbitrar. En efecto, existen en la actualidad muchas situaciones jurídicas en las que, para que una cláusula compromisoria produzca efectos reales, es necesario que esta abarque a más personas que aquellas que materialmente han firmado. Así, se trata de responder a la pregunta: ¿quiénes son verdaderamente partes de la cláusula compromisoria?

Si bien las cortes de diferentes países han establecido, en determinadas circunstancias, que el pacto arbitral abarca a más personas que aquellas que materialmente han firmado el contrato, prácticamente ninguna legislación interna ni ningún tratado internacional proporcionan criterios o herramientas para definir quiénes son parte en un acuerdo concreto (Born, 2014). Esto se torna especialmente difícil en la medida en que, por lo general, se requiere de una etapa probatoria que permita identificar a las personas que sí están vinculadas, lo que implica, necesariamente, emitir un laudo con el fin de determinar si una persona está o no vinculada. Por supuesto, tal situación es indeseable porque todo el proceso puede resultar inane si el tribunal llega a la conclusión de que el pacto no involucraba a una persona en concreto.

La teoría de la arbitrabilidad subjetiva distingue entre parte, no signatario y tercero siendo los dos primeros personas a quienes les es aplicable la decisión tomada por un tribunal arbitral, mientras que el último debe ser entendido como aquella persona que no puede ser vinculada por

del arbitraje en tanto algunos ordenamientos le dan un tratamiento al arbitraje como de naturaleza procesal. No obstante lo anterior, para efectos del presente trabajo se asumirá que, en todo caso, al analizar el pacto arbitral se está bajo un estudio que presume la condición de negocio jurídico del acuerdo de voluntades. 
un laudo (Gómez Londoño, 2013). Al respecto, debemos decir que el concepto de no signatario hace referencia a un claro tipo de parte, que es aquella cuya manifestación de la voluntad no se dio de manera expresa en el momento de la suscripción del acuerdo arbitral.

Ahora bien, luego de establecer algunas distinciones terminológicas y de delimitar el problema de estudio, es necesario pasar a evaluar y conceptualizar las diferentes posturas que se han desarrollado para responder al problema de la arbitrabilidad subjetiva.

\section{La figura de la estipulación por otro}

La figura de la estipulación por otro se produce en aquellos eventos en que "(...) aun cuando el tercero sea titular de un derecho derivado del contrato, no es parte" (Cárdenas, 2010).

De acuerdo con el profesor Larroumet (2004), la figura de la estipulación para otro constituye una excepción a la relatividad de los contratos, ampliando la arbitrabilidad subjetiva, en la medida en que se trata de un caso en el que un tercero del contrato puede llegar a ser vinculado.

El sustento filosófico detrás de que la estipulación por otro permita la vinculación de terceros a los contratos radica en la figura de la voluntad contractual dado que, si las partes han decidido celebrar un contrato que beneficia a un tercero, este se debe beneficiar en las estrictas condiciones en las que las partes decidieron otorgarle tal beneficio.

Sin embargo, no se puede perder de vista que el reconocimiento de la figura de la estipulación por otro, como una vía para hacer extensivo el pacto arbitral, no es universalmente reconocido puesto que, si bien jurisdicciones como las estadounidense, belga, alemana e italiana la han aceptado de forma general, no sucede igual respecto de la jurisdicción francesa (Clay, 2006).

La dificultad alrededor de esta perspectiva radica en el carácter voluntario del arbitraje en Colombia, así como en la problemática del arbitraje alrededor de la violación al derecho fundamental al acceso a la justicia. Por tanto, se hace fundamental el cumplimiento del requisito de que para que la estipulación por otro -el beneficiario o el patrimonio 
autónomo- genere efectos respecto del tercero, este debe prestar su consentimiento para participar 6 .

Entendemos que la estipulación por otro es una verdadera excepción al efecto relativo de los contratos en tanto su aplicación se realiza en función del estudio, caso a caso, de la situación. Por el contrario, aquellos procesos en los que la ley contempla como obligatoria la vinculación automática de un tercero al pacto arbitral, como es el caso de la sociedad aseguradora luego de la entrada en vigencia de la Ley 1563 de 2012, y, en consecuencia, se hace parte, en realidad no es una excepción al efecto relativo de los contratos, sino de una vinculación impuesta que se justifica en la aceptación del contrato accesorio (Mantilla Espinosa, inédito).

En esa medida, consideramos que se requiere la voluntad del tercero, pero no la validación de la voluntad de las partes que ya la habrán prestado por vía del reconocimiento de la celebración del contrato que constituye una estipulación en favor de otro. Así, podemos concluir que para que un beneficiario se haga parte de un trámite arbitral en virtud de una cláusula compromisoria, incluida en un contrato de fiducia, solo es necesaria su manifestación de la voluntad.

Siguiendo la lógica según la cual la voluntad de las partes es el elemento que justifica la ruptura del efecto relativo de los contratos, la vinculación del tercero debe estar limitada precisamente por el contenido específico de lo que las partes han acordado. En palabras del profesor Larroument (2004) "En la voluntad del promitente y el estipulante debe buscarse el origen y contenido de los derechos del beneficiario de la estipulación. Pues esos derechos no pueden ser más ni menos que los que ellos dos quisieron para el beneficiario". En consecuencia, en los casos en los que la vinculación del tercero se produce en virtud del beneficio que está adquiriendo por la estipulación por otro, deberá limitarse a la voluntad de los contratantes. 


\section{Perspectivas sobre la arbitrabilidad subjetiva y su aplicación frente a las operaciones fiduciarias}

El contrato de fiducia mercantil, de acuerdo con el Artículo 1226 del Código de Comercio es "un negocio jurídico en virtud del cual una persona, llamada fiduciante o fideicomitente, transfiere uno o más bienes especificados a otra, llamada fiduciario, quien se obliga a administrarlos o enajenarlos para cumplir una finalidad determinada por el constituyente, en provecho de este o de un tercero llamado beneficiario o fideicomisario". En esa medida, las partes del contrato son exclusivamente el fiduciante o fideicomitente y el fiduciario. Por tanto, a primera vista, parecería que estas dos personas son las que pueden: 1) estar facultadas para convocar la constitución de un tribunal arbitral; 2) participar de un proceso arbitral que se produzca como consecuencia del pacto arbitral; y 3) ser vinculadas por la decisión del tribunal arbitral.

Hoy en día se pueden identificar dos tendencias distintas que buscan explicar el problema de quien no es parte en el pacto arbitral incluido en el contrato de fiducia. En primer lugar, se encuentra una visión -que podemos llamar rígida- del vinculado por el pacto arbitral, según la cual solamente las partes del contrato podrán ser obligados por los efectos de un laudo proferido en virtud de un pacto arbitral. En segundo lugar, hay una visión flexible de la arbitrabilidad subjetiva conforme a la que, aquel que se beneficia del contrato, adquiere tal provecho en las condiciones que inicialmente fueron acordadas por las partes $y$, en consecuencia, estas incluyen los requisitos en los que se produce tal beneficio, incluyendo, por ejemplo, el sistema de solución de controversias que se haya fijado.

La visión rígida es aquella que restringe los efectos del pacto arbitral exclusivamente a las personas que de manera formal figuren como partes del contrato en el que se encuentra incorporado el pacto arbitral sin excepción alguna. Se entiende como rígida porque no busca de ninguna manera analizar la verdadera situación jurídica del caso. En otras palabras, asume que las partes del pacto arbitral, en su sentido procesal, coinciden con aquellas personas que son parte del contrato que contiene dicho pacto. Es quizá la posición que, en general, tiene más acogida $\mathrm{y}$, probablemente, la respuesta que otorga mayor seguridad jurídica por 
cuanto da certeza de que un laudo solo afectará a aquel que haya dado su consentimiento como parte.

Como lo hace notar Sinisterra Páez (2010), las principales definiciones de pacto arbitral contenidas en las legislaciones internas y en los instrumentos internacionales hacen parte de la categoría que hemos llamado visión rígida. Entre muchos ejemplos legislativos de estas características se pueden mencionar el Artículo II de la Convención de Nueva York, cualquiera de las opciones incorporadas en el Artículo VII de la Ley Modelo de la Comisión de las Naciones Unidas para el Derecho Mercantil Internacional, la Convención Interamericana de Arbitraje Internacional, así como las definiciones de pacto arbitral que se encuentran incorporadas en la legislación interna de España ${ }^{7}$, Perú $^{8}$, Chile 9 y Francia ${ }^{10}$, entre otros. En el mismo sentido, la Ley 1563 de 2012, en su Artículo $1^{\circ}$ señala que: "El arbitraje es un mecanismo alternativo de solución de conflictos mediante el cual las partes defieren a árbitros la solución de una controversia relativa a asuntos de libre disposición o aquellos que la ley autorice". Por tanto, la posición que acoge la legislación nacional sostiene que la competencia ratio personae se limita a quienes son parte del pacto arbitral.

En el mismo sentido, y en aplicación de un caso relativo a un contrato de fiducia, la Sala de Casación Civil de la Corte Suprema de Justicia, en sentencia del $1^{\circ}$ de julio de 2009, parece dar respuesta a este inquietud al analizar una situación en la que la sociedad Weston Ltda. (beneficiario)

7 El Artículo 9 (1) de la Ley de Arbitraje española, Ley 60 de 2003, señala que "El convenio arbitral, que podrá adoptar la forma de cláusula incorporada a un contrato o de acuerdo independiente, deberá expresar la voluntad de las partes de someter a arbitraje todas o algunas de las controversias que hayan surgido o puedan surgir respecto de una determinada relación jurídica, contractual o no contractual".

8 La ley general de arbitraje de Perú, Decreto Legislativo 1071 de 2008, señala que "El convenio arbitral es un acuerdo por el que las partes deciden someter a arbitraje todas las controversias o ciertas controversias que hayan surgido o puedan surgir entre ellas respecto de una determinada relación jurídica contractual o de otra naturaleza".

9 El Artículo 7 (1) de la Ley de Arbitraje, Ley 19.971, señala que "El acuerdo de arbitraje es un acuerdo por el que las partes deciden someter a arbitraje todas las controversias o ciertas controversias que hayan surgido o puedan surgir entre ellas respecto de una determinada relación jurídica, contractual o no contractual".

10 El Artículo 1442 del Nuevo Código de Procedimiento Civil señala que "La clause compromissoire est la convention par laquelle les parties à un contrat s'engagent à soumettre à l'arbitrage les litiges qui pourraient naitre relativement à ce contrat". 
demandó a las sociedades Cipres Trade Center S. A. (fideicomitente) y Fiduciaria Santander S. A. en liquidación (fiduciante) por los perjuicios ocasionados como consecuencia de un contrato de promesa de compraventa entre el demandante y Cipres Trade Center S. A., para la venta de locales de un centro comercial que sería construido con recursos que administraría la sociedad fiduciaria mencionada.

El cargo primero de la demanda de casación formulada - relevante para el presente artículo- fue la existencia de un pacto arbitral de manera que el juez estatal, a juicio del actor, carecía de jurisdicción. La corte desestima tal postura en el entendido de que Weston Ltda. es un tercero respecto del contrato de fiducia y que, por tanto, no puede ser considerado como parte obligada por el pacto arbitral que se incluía (Corte Suprema de Justicia, 2009).

Si bien en el caso estudiado el resultado fue favorable para el beneficiario, la corte presenta una posición según la cual solamente las partes del contrato podrían activar la competencia del tribunal arbitral y, por tanto, el beneficiario no estaba legitimado para hacerlo sino que, necesariamente, debía recurrir a la jurisdicción nacional. Dicha postura implica que existan problemas de competencia en la medida en que, una vez se inicie el proceso, surgirán las excepciones de la cláusula arbitral -como sucedió de forma tardía en el caso sub judice- o, peor, implicará que, en tales casos, la voluntad de las partes manifestada por medio del pacto arbitral no tendrá resultados prácticos, sino que las controversias serán resueltas por jueces estatales.

El tribunal precisó el estatus del beneficiario de una fiducia mercantil al sostener que:

Por definición legis, las partes del negocio jurídico de fiducia mercantil, son el fiduciante o fideicomitente y el fiduciario; el beneficiario o fideicomisario, expressis verbis es "un tercero", cuya previsión por las partes, ni siquiera es menester para la celebración, existencia o eficacia final de la fiducia mercantil. En cuanto la finalidad fiduciaria podrá determinarse por el constituyente, fiduciante o fideicomitente, exclusivamente en su provecho, y determinada, por este, a favor de un tercero, no por ello adquiere la calidad de parte, aunque su existencia condiciona la eficacia final del acto dispositivo, sus efectos definitivos, los fines perseguidos 
por las partes y su función práctica o económica social (Sentencia de casación, Corte Suprema de justicia, 2009).

Si se toma entonces la posición presentada y se hace la respectiva aplicación al problema de la arbitrabilidad subjetiva, se concluye que: 1) tan solo podrán ser parte del tribunal arbitral el fideicomitente y el fiduciario; y 2) las decisiones tomadas por parte del tribunal arbitral solo podrán referirse a los derechos de los que sean titulares el fideicomitente y el fiduciario. Se infiere entonces, de semejantes premisas, que un pacto arbitral no tendría ninguna efectividad en el caso de los contratos de fiducia.

Es importante notar que esta posibilidad no es para nada ajena al sistema jurídico colombiano que contempla, en el Artículo 1903 del Código Civil, al regular la garantía por evicción en la compraventa, que la obligación de sanear cesa si el comprador y quien demanda la cosa como suya es sometida a arbitraje sin el consentimiento del vendedor y los árbitros fallaren contra el comprador.

La Corte Constitucional colombiana parece tener la misma postura al respecto pues tiende, ante todo, hacia la exaltación del principio de habilitación (Gómez Londoño, 2013) según el cual el tribunal arbitral solamente está habilitado para decidir sobre derechos de los que sean titulares las personas que fueron signatarias del pacto arbitral.

Tal posición se evidenció en la Sentencia C-170 de 2014 al analizar la vinculación de terceros en procesos arbitrales, sobre todo respecto del Artículo 37 de la Ley 1563 de 2012, de manera que estableció una serie de criterios que sugerirían que el beneficiario del contrato de fiducia no está en capacidad de activar la composición de un tribunal arbitral cuya cláusula compromisoria se encuentre en el contrato de fiducia. La corte sostiene, al analizar las maneras de vincular a un tercero en calidad de llamamiento en garantía, que:

(...) la norma acusada garantiza plenamente que la jurisdicción arbitral se active únicamente por habilitación expresa de las partes. Por ello, se reitera, el sentido del parágrafo demandado, indica que el tercero garante afectado de manera "automática" es aquel que conoció el contrato que se obligó a garantizar y que incluía pacto arbitral voluntario 
sustentado en la autonomía de quienes lo suscribieron. (...) Por ello, lo que realmente vulneraría el principio de voluntariedad de la jurisdicción arbitral, sería permitirle al tercero que desconozca la manifestación de dos voluntades autónomas para acudir a árbitros y no a jueces de la República (Sentencia C-170 de 2014).

Esta posición de la corte respecto del tercero llamado en garantía ilustra que la capacidad de iniciar un proceso arbitral está exclusivamente en cabeza de las partes del contrato y, por tanto, no faculta al beneficiario ni a ningún otro tercero para activar la jurisdicción del tribunal, lo que lo pone en desventaja.

A pesar de que se trate de la posición preponderante, son evidentes las implicaciones negativas que tiene la visión rígida del pacto en la medida en que una posición jurídica que restrinja la vinculación absoluta de personas no signatarias redunda en la práctica en soluciones contrarias a la voluntad de las partes. Lo anterior si, como resultado de la exclusión del tercero del pacto arbitral, todas las controversias que este quisiera iniciar terminaran en la jurisdicción ordinaria a pesar de que las partes hubieran pactado la solución de sus controversias por medio de la justicia arbitral.

Como ya se mencionó, el argumento a favor de la visión rígida de la arbitrabilidad subjetiva es la seguridad jurídica. Por el contrario, en una visión que llamamos flexible, el gran riesgo es la vinculación a un laudo arbitral de personas que no hayan manifestado su voluntad de someter las controversias a este tipo de justicia heterocompositiva. Por el otro lado, la característica más beneficiosa de esta teoría radica en que se genera un análisis de las circunstancias reales del vínculo jurídico para establecer quién está obligado por el pacto arbitral. La llamamos flexible porque constituye una excepción al principio de relatividad de los contratos en tanto admite que terceros, que no hacen parte del contrato, sean vinculados por el mismo.

El caso de Dallah Real Estate and Tourism Holding Company contra el gobierno pakistaní es un ejemplo de un análisis que permitió la vinculación de personas que no eran parte en un sentido formal del contrato, pero que igual debían ser obligadas por la decisión tomada por el tribunal arbitral. Someramente se pueden resumir los hechos en que Dallah suscribió un contrato con un Patrimonio Autónomo, creado por el gobierno 
de Pakistán, que tenía por finalidad proveer hospedaje a los peregrinos pakistaníes que quisieran viajar a La Meca. Dentro del contrato se pactó la posibilidad de que el Patrimonio Autónomo cediera los derechos y obligaciones al Estado de Pakistán, así como la cláusula arbitral en caso de suscitarse una controversia. Adicionalmente, el gobierno de Pakistán sería el garante dentro del contrato. En desarrollo del mencionado negocio jurídico, el gobierno de turno de Pakistán, del año 1996, optó por no continuar con el fideicomiso, lo que desencadenó la acción por parte de Dallah. El gobierno propuso como argumento central de su defensa que no era parte del contrato, por lo que no podía ser citado al Tribunal de Arbitramento. En el desarrollo del litigio, Dallah obtuvo laudo a favor en el que se condenaba al Estado Pakistaní por 20.588,040 dólares estadounidenses. Posteriormente, elevó dos solicitudes de exequatur que fueron resueltas en sentido contrario, aun cuando el sustento era la misma ley francesa.

El tribunal inglés, aplicando la ley francesa, sostuvo que el Estado pakistaní, aun no siendo parte dentro del contrato celebrado en su oportunidad por Dallah y el Patrimonio Autónomo, estaba vinculado por la cláusula arbitral, toda vez que la intención de los contratantes - esto es, de las partes en el contrato- había sido la de darle fuerza vinculante respecto del gobierno de Pakistán. Por otro lado, aplicando también la ley francesa, la solicitud de exequatur ante el tribunal francés arrojó un resultado distinto. En efecto, este sostuvo que el gobierno pakistaní no era parte dentro del contrato y que, de acuerdo al antecedente del caso Orri vs. Societé des Lubrifiants Elf Aquitaine (1992), la costumbre mercantil internacional estableció que la cláusula arbitral se podía extender a no signatarios del contrato cuando se cumplieran dos requisitos: 1) que estuviera directamente relacionada con la ejecución del contrato; y 2) que de las relaciones comerciales existentes entre las partes se presumiera que el no contratante aceptaría el arbitraje por saber de su existencia y de su alcance. Consideramos que se trata de una excepción al efecto relativo de los contratos en tanto, en el caso bajo estudio, no existe un consentimiento respecto de la vinculación al pacto arbitral sino, simplemente, un conocimiento de la existencia del mismo respecto de una persona que no es parte en el contrato celebrado. En conclusión, ambos tribunales, en la temática que nos atañe, llegan a la misma conclusión: sí puede haber 
personas vinculadas por un pacto arbitral incluido en un contrato de trust, independientemente de que no sean consideradas partes en el contrato.

En el mismo sentido, la figura del no signatario tiene por objeto la vinculación a un proceso arbitral a personas que en virtud de las circunstancias de la relación comercial relevante sí deben ser vinculados (Park, 2009).

De la misma manera, la jurisprudencia estadounidense ha decantado que, si bien el pacto arbitral es producto de la voluntad de las personas, un no signatario del pacto podrá ser vinculado a un proceso arbitral si ello se desprende de los principios generales de los contratos y las leyes de agencia (Lee Eisen, 2001). Tal postura hace parte de lo que hemos denominado una visión dinámica de la arbitrabilidad subjetiva puesto que no limita a un análisis exclusivamente normativo sino, sobre todo, fáctico de la realidad económica y jurídica que subyace al contrato para lograr una verdadera solución eficaz y respetuosa de la voluntad de las partes.

Ahora bien, esta teoría impone un papel más activo por parte del juez del caso. De hecho, llama la atención que sea una figura que se haya desarrollado en el sistema del common law, en el que el juez cumple con un papel protagónico. El problema radica en que el activismo del juez en dilucidar la voluntad de las partes se traduce en interpretaciones que no fueron su voluntad y que, por tanto, se vincule a terceros absolutos confundiéndolos con no signatarios.

Existen otras situaciones en las que existe mayor desarrollo sobre el asunto. Así, por ejemplo, en los casos en los que el vínculo que justifica el interés del tercero es anterior a la constitución de la fiducia, no hay duda respecto de su capacidad para participar del proceso arbitral. El profesor Álvarez Gómez (1993) sostiene, al mencionar el problema de la vinculación del patrimonio autónomo en procesos contra el fideicomitente, que: "En este orden de ideas, nos parece claro que cualquier discusión que se presente entre un acreedor del fiduciante anterior al contrato de fiducia, para la solución de la obligación a través del ejercicio del derecho sobre los bienes fideicomitidos, impone la presencia en el proceso como demandado, del patrimonio autónomo y, lógicamente, de la sociedad fiduciaria en representación de aquel, para atender los intereses de este", no parece que exista motivo alguno por el que lo propio no pueda ser predicado respecto de procesos arbitrales. 
Ahora, es necesario, a la luz de casos prácticos, estudiar las diferentes soluciones que estas posturas pueden implicar.

\section{Problemas prácticos de la arbitrabilidad subjetiva en el contrato de fiducia: un riesgo latente}

Con respecto al contrato de fiducia mercantil, con la presencia del fideicomitente y de la sociedad fiduciaria es suficiente para constituir dicho contrato y crear un patrimonio autónomo que cuenta con un estatuto jurídico particular por medio del que está en capacidad de tener obligaciones y derechos independientes de los de su fideicomitente y de la sociedad fiduciaria, aunque esta última deba actuar como su vocera, con lo que no cabe duda de su condición de parte tanto a nivel sustancial, como a nivel procesal ${ }^{11}$. Si a lo anterior se agrega la figura del beneficiario -que puede concurrir con la figura de fideicomitente y beneficiario-, se está ante la tipología clásica del contrato como lo muestra la figura 1.

Figura 1. Tipología clásica del contrato de fiducia

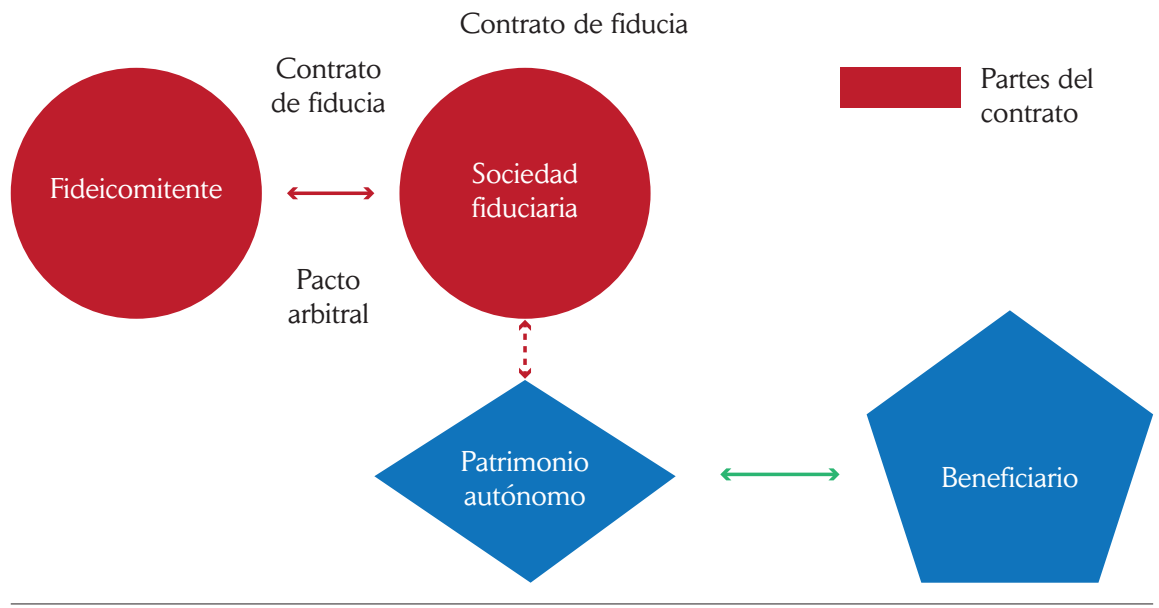

Fuente: elaboración propia.

11 Consejo de Estado. Sección tercera. Sentencia del 25 de septiembre de 2013. M. P. Mauricio Fajardo Gómez. Exp. 19.933. 
Es claro entonces que ni el patrimonio autónomo, como sujeto que puede ser titular de derechos y obligaciones, ni el beneficiario son partes en el contrato de fiducia. Respecto del patrimonio autónomo, su calidad en cuanto al contrato se torna irrelevante por cuanto la sociedad fiduciaria, de cuya calidad de parte no hay ninguna duda, es su vocera.

Sin embargo, es posible contemplar la existencia de otro tipo de terceros que son quizás aún más distantes del negocio fiduciario, pero que pueden estar interesados en participar en procesos arbitrales en los que se ventilen intereses y derechos propios como consecuencia de vínculos jurídicos con el patrimonio autónomo. Teniendo en cuenta lo anterior, se contemplará, como criterio diferenciador para analizar diferentes hipótesis que pueden surgir, el tipo de persona que está vinculada, o mejor, el papel contractual que juega.

En primer lugar, se estudiará el caso del fideicomitente que es a la vez tercero de un contrato que el patrimonio autónomo celebra en el marco de la ejecución de las actividades que desempeña. En segundo lugar, se abordarán los casos en los que es un tercero quien está interesado o vinculado al pacto arbitral. En tercer lugar, se contemplará la posición del beneficiario cuando este coincide en cabeza del fideicomitente. Finalmente, en cuarto lugar, cuando el beneficiario, al mismo tiempo, tiene un contrato con el fideicomitente.

\section{La vinculación de quien ostenta simultáneamente el carácter de tercero y fideicomitente}

En principio, si un tercero celebra un contrato con el patrimonio autónomo, este es absolutamente independiente del contrato de fiducia al punto de que, en realidad, al tercero le podría ser irrelevante cualquier tipo de conflicto que pudiera surgir entre el fideicomitente y la sociedad fiduciaria. Sin embargo, la situación puede ser diferente en el caso en el que el fideicomitente sea a la vez un tercero que contrata con el patrimonio autónomo que se constituyó como consecuencia del contrato de fiducia mercantil. La figura 2 muestra cómo concurren las dos figuras en la misma persona de lo que puede surgir la pregunta de si las cláusulas arbitrales, entre cada uno de los contratos, pueden llegar a ser unidas a efectos de que el tribunal pueda tomar decisiones con base en 
la totalidad de las relaciones comerciales. Sin embargo, es claro que los extremos de ambos pactos arbitrales no son los mismos. Respecto del contrato de fiducia se trata de la sociedad fiduciaria, mientras que en el caso del contrato adicional, las partes serán el fideicomitente y el patrimonio autónomo al margen de que su vocero sea la sociedad fiduciaria.

Figura 2. Contrato con fideicomitente co-contratante

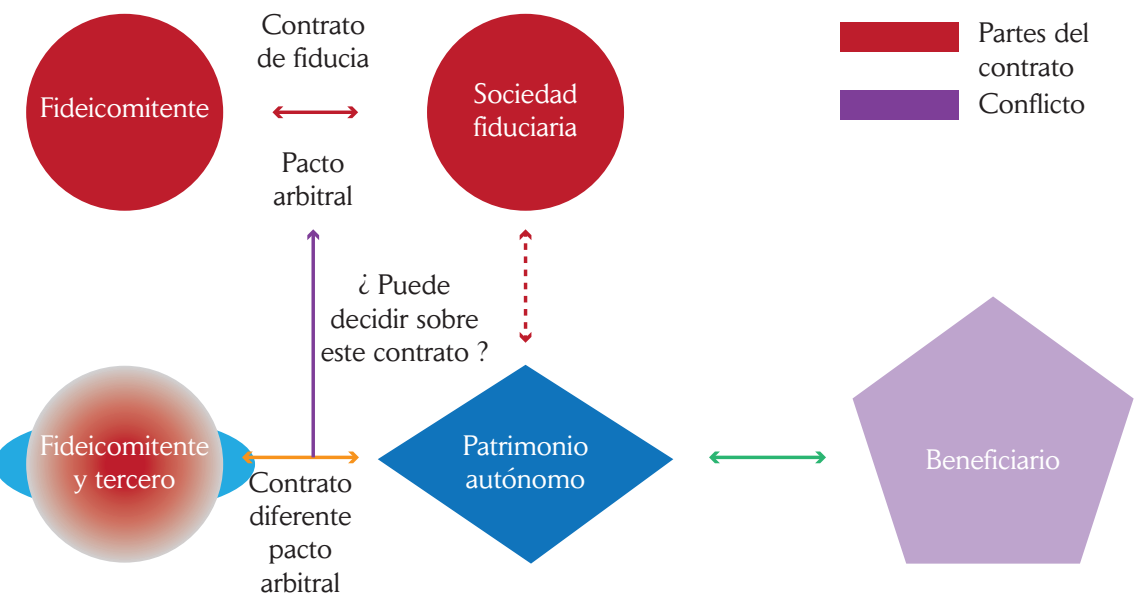

Fuente: elaboración propia.

El caso de la hipótesis mencionada ya se dio y fue tratado por la jurisprudencia arbitral en laudo con fecha de 30 de agosto de 2012 -árbitro único Luis Carlos Gamboa- en el caso Patrimonio Autónomo Fideicomiso Pagos Procampo (en adelante B) frente a una sociedad denominada Productividad Para el Campo S. A. -Procampo S. A. en concordato, Productos Agropecuarios Ltda. y Cía. en concordato y Química Moderna S. A.- Quimor S. A. en concordato (en adelante A). A constituyó una fiducia con la sociedad fiduciaria $B$, quien a su vez celebró un contrato de arrendamiento, en calidad de vocera de los bienes fideicomitidos, con A. En el segundo contrato se incorporaron como obligaciones del arrendatario el pago de un canon, así como el pago del impuesto de timbre correspondiente. Tras un incumplimiento de los cánones, la fiduciaria inicia el proceso arbitral de acuerdo con el pacto arbitral incorporado dentro del contrato de arrendamiento. La sociedad A, por su parte, realiza una demanda de reconvención solicitando que se reconozca la ilicitud del 
contrato por medio del cual B buscaba evadir el proceso de liquidación obligatoria de la sociedad, empleando la figura del acuerdo de recuperación de los negocios de las deudoras. En la contestación de la demanda de reconvención, la fiduciaria $\mathrm{B}$ sostiene que el tribunal carece de competencia para conocer del contrato de fiducia bajo el entendido de que este cuenta con una cláusula compromisoria autónoma. El árbitro único, al momento de manifestarse respecto de la competencia del tribunal, hace referencia a dos elementos relevantes para efectos del presente artículo: 1) sostiene que, al interpretar la cláusula arbitral, ella implica el análisis sobre la validez del pacto arbitral y, por tanto, la causa del contrato de arrendamiento y su licitud deben ser tenidas en cuenta por el tribunal; y 2) como el pacto arbitral se circunscribe al contrato de arrendamiento, el árbitro no se puede pronunciar respecto de elementos propios del contrato de fiducia, ni de ningún otro contrato que le corresponda.

La decisión que tomó el árbitro demuestra las limitaciones que este tiene a la hora de evaluar las relaciones contractuales de forma holística. Claramente, las condiciones de negociación del contrato de arrendamiento, e incluso su propio incumplimiento, no pueden ser desligadas del vínculo que existía de forma previa por medio del contrato de fiducia mercantil. No parece que las limitaciones que impone la teoría de la relatividad de los contratos pueda explicar de forma acertada el fenómeno en el caso sub judice puesto que, al parecer, la voluntad de las partes sí era arbitrar las controversias de los dos contratos de manera que la forma más conveniente hubiera sido la unificación de las cláusulas arbitrales. Sin embargo, esta posibilidad no puede ser contemplada si se considera que las partes en ambos contratos no son los mismas. El laudo estudió de forma somera las condiciones del contrato de fiducia -aunque no se pronuncia sobre su ilicitud- bajo el argumento de que ello se desprendía del elemento causa del contrato de arrendamiento; de lo contrario, no habría podido abordar de ninguna manera el contrato entre el fideicomitente y el fiduciario.

\section{La vinculación de un tercero}

A pesar de las complejidades ya expuestas del caso anterior, surge otra hipótesis: ¿qué sucede si el tercero no es, a la vez, el fideicomitente? 
¿Qué hubiera sucedido si, en el caso anterior, el contrato de arrendamiento hubiera sido celebrado con un tercero, ajeno al contrato, pero que de igual forma identificase alguna causal de ilicitud del contrato que constituyó al patrimonio autónomo con el que hubiera contratado o que percibiera una irregularidad en el mismo que implicara, en algún momento, la nulidad del contrato? Así mismo, como lo muestra la figura 3, surgen de esta hipótesis dos escenarios diferentes: la participación del tercero en el proceso arbitral que surgiera del pacto arbitral incluido en el contrato de fiducia y la participación del fideicomitente en el contrato en el que el tercero fuera parte ante las irregularidades en el ejercicio de la actividad de la sociedad fiduciaria.

Figura 3. Negocios jurídicos con terceros absolutos

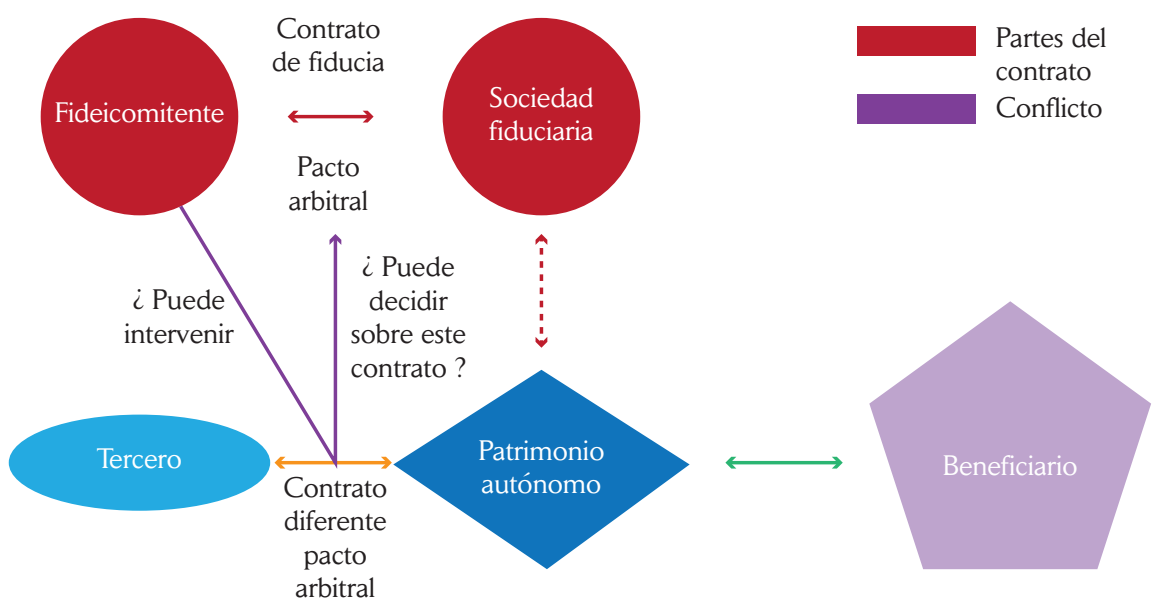

Fuente: elaboración propia.

Respecto de la segunda hipótesis, no hay grandes complicaciones en la medida en que el fideicomitente no podrá, de ninguna manera, interferir en el proceso que se genere como consecuencia del pacto arbitral. Sin embargo, podrá tener acceso al pago de perjuicios por parte de la sociedad fiduciaria si su actividad no es adecuada o es violatoria de alguno de los deberes y obligaciones que le corresponde legal y contractualmente. Por el contrario, en la primera hipótesis, el tercero se encuentra desprotegido en su situación, motivo por el que se debe encontrar un 
mecanismo para que pueda participar y garantizar la protección de sus derechos. En definitiva, la razón que justifica la ruptura del efecto relativo de los contratos en dicho caso es que el tercero se esté beneficiando de tal posibilidad, sin que ello traiga como consecuencia ninguna obligación automática, sino solamente aquellas que se derivarían de su manifestación en el sentido de adherir al pacto arbitral.

Así mismo, el problema de la vinculación al proceso de un no signatario no implica que se solucione la incapacidad del mismo para convocar la constitución del tribunal arbitral. En el presente caso, ya es poco probable que sea posible la vinculación del tercero en el proceso arbitral; sin embargo, todavía será menos probable que el tercero pueda iniciar el proceso con base en el pacto arbitral celebrado por las partes, en la medida en que la legislación colombiana ni siquiera otorgará el carácter de no signatario, sino de tercero.

\section{La vinculación del beneficiario cuando este es el fideicomitente}

La participación del beneficiario, cuando coincide con la figura del fideicomitente, es un problema de orden teórico, mas no práctico. Ciertamente el beneficiario tendrá las mismas dificultades que el tercero para poder alegar su participación dentro del proceso arbitral. Sin embargo, por su calidad de fideicomitente, podrá iniciar el proceso en calidad de parte sin tener ningún impedimento en materia de arbitrabilidad subjetiva.

\section{La vinculación del beneficiario}

Cuando el beneficiario es completamente ajeno a las figuras de las partes del contrato, este ostenta - de acuerdo con la ley colombiana- el carácter de tercero, como lo grafica la figura 4 . En ese sentido, a la luz de que la tendencia principal de la Corte Constitucional de Colombia ha sido por el principio de la habilitación (Gómez Londoño, 2013), según el cual el tribunal solamente está habilitado para decidir sobre derechos de los que sean titulares las personas que fueron signatarias del pacto arbitral, pareciera que el beneficiario no tiene ninguna posibilidad de intervenir o de convocar el tribunal arbitral respecto del contrato de fiducia, aun 
cuando puede ser este el más interesado en la apropiada solución de la controversia.

Figura 4. E1 beneficiario como tercero absoluto

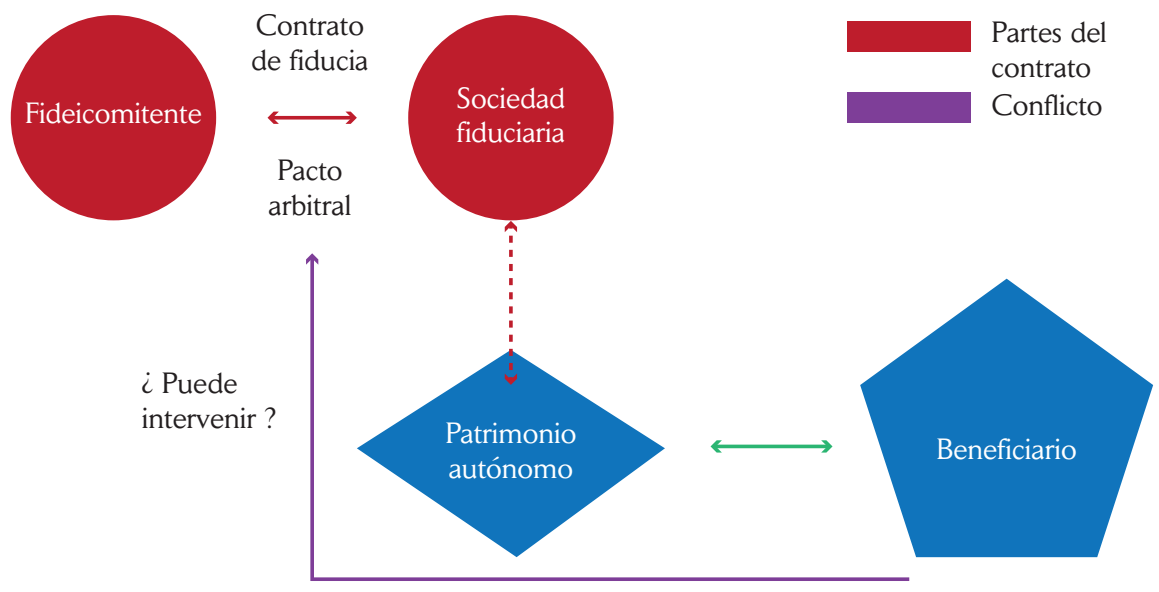

Fuente: elaboración propia.

La incapacidad del beneficiario de poder hacer uso del pacto arbitral redunda, en últimas, en un defecto de la figura que se puede traducir en desconfianza o insatisfacción. Si el interés a nivel global es la construcción de figuras jurídicas que puedan ser empleadas por su confiabilidad, muy seguramente el beneficiario, aun cuando no tiene ningún otro vínculo, debería estar mejor protegido y amparado de aquella manifestación de la voluntad de las partes según la cual los conflictos serían solucionados por medio de arbitraje.

\section{La vinculación del beneficiario cuando hay un contrato de garantía}

A diferencia de la situación que se mencionó en el numeral anterior, las circunstancias cambian en el caso en el que entre el fideicomitente y el beneficiario existen otros vínculos comerciales. El ejemplo quizá más apropiado es, precisamente, el de la fiducia en garantía, en la medida en que, precisamente, el beneficiario celebra un contrato con el fideicomitente que será garantizado por parte de un patrimonio que se crea como consecuencia de un contrato celebrado entre la sociedad fiduciaria y el 
fideicomitente del que el beneficiario es un tercero, como lo explica la figura 5 .

Figura 5. E1 beneficiario como tercero y como co-contratante

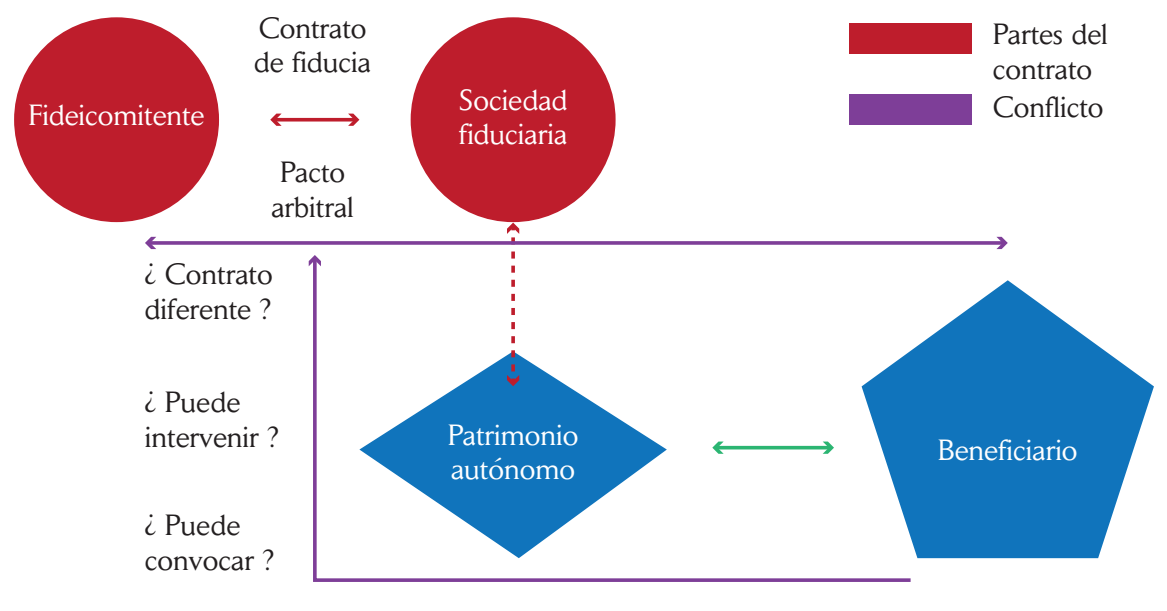

Fuente: elaboración propia.

Tribunales arbitrales colombianos ya han decidido casos que se pueden encontrar dentro de este supuesto. Infortunadamente, las decisiones tomadas han sido contradictorias entre sí.

En el caso Leasing Mundial S. A. contra Fiduciaria FES S. A., de agosto 26 de 1997 (árbitros Jorge Suescún Melo, quien preside, Jorge Cubides Camacho y Antonio Aljure Salame), la sociedad B celebró un contrato de fiducia mercantil irrevocable de garantía con la sociedad fiduciaria C, contrato del que la sociedad D era beneficiaria. El contrato contenía una cláusula arbitral vinculante para fideicomitente, fiduciario y beneficiario. El beneficiario inicia el proceso arbitral por cuanto $C$ emitió un certificado de garantía fiduciaria a pesar de que no se había constituido un patrimonio autónomo. La sociedad B entra en un proceso de concordato, motivo por el cual sus bienes son embargados, incluyendo a los que debían ser parte del patrimonio autónomo puesto que la escritura pública correspondiente al contrato de fiducia nunca fue registrada. El tribunal, al momento de analizar la cláusula arbitral, manifiesta que se trata de un ejemplo de estipulación a favor de otro, figura que se encuentra en el Código Civil colombiano, en el Artículo 1506, que supone 
que debe haber una aceptación por parte del tercero involucrado en un contrato que no suscribió. El tribunal encontró probada tal circunstancia en el momento en el que aceptó la recepción del certificado de garantía puesto que una cláusula del mismo así lo manifestaba.

Quizá la mayor dificultad que atraviesa una figura en tales condiciones es la posición contractual del beneficiario que establece, de forma expresa, que se trata de un tercero. Si bien el tribunal arbitral acoge la postura que luego será asumida por la Corte Suprema de Justicia -la de la estipulación a favor de un tercero, supedita la participación del beneficiario a la correcta redacción de la cláusula arbitral, al margen del hecho de que sostiene que, sin importar la denominación que las partes le otorguen, el beneficiario siempre será un tercero.

En el caso Comercial Patrimonio S. A. contra Fiduanglo S. A., la sociedad fiduciaria había realizado un contrato de fiducia en garantía con Procampo S. A. y Proagro Ltda. y Cía. Ltda. S. en C. S., del que Comercial patrimonio S. A. era beneficiario. El contrato incluía una cláusula arbitral que manifestaba vincular al fideicomitente y al fiduciario. El tribunal arbitral, convocado por el beneficiario, niega su competencia con el siguiente argumento:

La calidad de parte surge de la naturaleza misma del negocio jurídico de que se trate y de la actuación del sujeto en cuestión. Y en el contrato de fiducia y, más concretamente de fiducia para garantía de acreencias $\mathrm{y}$ acreedores por designar, son partes el fiduciante o fideicomitente y el fiduciario, exclusivamente. Los beneficiarios de la fiducia, aquí "acreedores garantizados", sean ya acreedores o lleguen a serlo luego, no son parte en el contrato son, como su propia denominación lo indica y lo impone la función económico-social de la figura legis, terceros beneficiarios, y no parte. Esto independientemente de si en el contrato se les llama "partes", pues los contratantes no podían desarticular la esencia del contrato para convertirlos en tales, (...) En cuanto al pacto arbitral propiamente dicho, es nítida y terminante la exigencia de que se celebre por escrito, con manifestación expresa de quienes lo acuerdan, de "su propósito de someterse a decisión arbitral". Naturalmente, someter ellos las disputas entre sí, y no las que puedan suscitarse entre cualquiera de ellos, o ambos, y un tercero. El pacto arbitral implica un asentimiento 
de cada cual de los interesados, con ámbito determinado, objetivo y personal (Comercial patrimonio S. A. contra Fiduanglo S. A., 2000).

Así mismo, sostiene que la interpretación del contrato permite concluir que la voluntad común era revestir al beneficiario de las prerrogativas propias de las partes del contrato, para lo que debía producirse la aceptación del mismo. En ese sentido, el tribunal no encuentra probada tal aceptación.

\section{Conclusiones}

Teniendo en cuenta todo lo que se ha analizado en el presente artículo, es posible concluir que las soluciones planteadas por la jurisprudencia colombiana no revisten de la riqueza teórica suficiente para garantizar la protección de los derechos de terceros en algunos casos, en particular para garantizar los derechos del beneficiario. En esa medida, es necesario abordar los problemas de las cláusulas arbitrales en contratos de fiducia desde una perspectiva mucho más detallada.

Encontramos, además, que para dar un mejor tratamiento al problema planteado es necesario hacer mayor claridad conceptual a las figuras de parte contractual y procesal, así como las de parte, parte no signataria y tercero, que son cruciales para el recto entendimiento del problema de la arbitrabilidad subjetiva.

En nuestro concepto, la aproximación, asumida recientemente, de solucionar los problemas que atraviesa el beneficiario por vía de la figura de la estipulación por otro no parece ser suficiente y genera inseguridad jurídica puesto que se somete a un altísimo margen de discrecionalidad por parte del juez o árbitro. De hecho, tal figura abre camino para un evento que aún no se ha visto, pero que es perfectamente viable, esto es, la anulación de laudos arbitrales por parte del juez de control bajo el argumento de inexistencia del pacto arbitral.

Mencionado el riesgo, nos permite concluir que, hasta tanto no se corrijan tales dificultades teóricas, en la práctica el arbitramento no será utilizado con la frecuencia necesaria. Así mismo, debemos concluir que los diversos criterios y posiciones que han sido adoptados dificultan la 
capacidad de predecir el resultado de incorporar cláusulas compromisorias a contratos de fiducia, lo que, de nuevo, desincentiva la utilización de este medio de solución de controversias.

De la revisión doctrinal y de la jurisprudencia extranjera se pueden desprender dos vías para la solución de los problemas enunciados: en primer lugar, existe una solución que pueden abordar las partes, relacionada con la redacción de cláusulas compromisorias que aclaren la difusa situación del beneficiario y, en algunos casos, de otros terceros que puedan estar interesados en participar en calidad de parte procesal dentro del tribunal de arbitramento.

En segundo lugar, los problemas detectados en este artículo pueden ser mitigados por vía del reconocimiento legal y jurisprudencial de que la participación del beneficiario es un verdadero caso de ruptura del principio de la relatividad de los contratos que se ve justificado por el derecho al acceso a la justicia que, de otra forma, se ve profundamente afectado.

\section{Referencias}

Alvaréz Gómez, M. A. (1993). La fiducia mercantil y el derecho de los acreedores sobre los bienes fideicomitidos. Revista de derecho privado, 13, 1-37.

Asofiduciarias. (2014). Informe anual año 2013. Recuperado de https://www. asofiduciarias.org.co/images/informe/Informe\%20Anual $\% 20$ Sector $\% 20$ Fiduciario\%202013.pdf

Born, G. (2014). International commercial arbitration: International and USA aspects commentary and materials. Londres: Kluwer Law International.

Caivano, R. (2006). Arbitraje y grupos de sociedades. Extensión de los efectos de un acuerdo arbitral a quien no ha sido signatario. Recuperado de http:// www.limaarbitration.net/LAR1/roque_j_caivano.pdf

Caivano, R. (2007). Algunos problemas derivados de los arbitrajes con partes o relaciones jurídicas múltiples. Revista Peruana de Arbitraje, IV. Recuperado de http://www2.congreso.gob.pe/sicr/cendocbib/con4_uibd.nsf/6444DDE2BE6 C54EE05257D070058D796/\$FILE/Revista_Peruana_Arbitraje_4_2007.pdf

Cárdenas, J. (2010). El efecto relativo de los contratos, su desarrollo y sus proyecciones. En Universidad Javeriana (ed.), Colombia, Realidades y tendencias del derecho en el siglo XXI (pp. 411-444). Bogotá: Temis. 
Cárdenas, J. (2014). El contrato de fiducia y el patrimonio autónomo. Bogotá: Editorial Universidad del Rosario.

Clay, T. (2006). La extensión de las cláusulas compromisorias a las partes nosignataria (fuera de los grupos de contratos y grupos de sociedades). Revista de la Corte Española de Arbitraje, 13-22.

Congreso de Colombia. ( 12 de julio de 2012). Código General del Proceso. Ley 1564 de 2012. Do. 48.489.

Congreso de Colombia. ( 12 de julio de 2012). Estatuto Nacional e Internacional de Arbitraje. Ley 1563 de 2012. Do. 48.489.

Congreso de Colombia. (31 de mayo de 1873). Código Civil colombiano. Ley 84 de 1873. Do. 2.867

Consejo de Estado. Sección Tercera. (25 de septiembre de 2013). Exp. 19.933. M. P. Mauricio Fajardo Gómez.

Corte Constitucional, Sala Plena. (19 de marzo de 2014). Sentencia C-170 de 2014. M. P. Alberto Rojas Ríos.

Corte Constitucional. Sala Plena. (14 de marzo de 2007). Sentencia SU-174 de 2007. M. P. Manuel José Cepeda Espinosa.

Corte Suprema de Justicia de Colombia. Sentencia de casación. (1 de julio de 2009). 11001-3103-039-2000-00310-01. M. P. William Namen Vargas.

Cour d'Appel, Paris, First Pole, First Chamber (17 febrero de 2011). Government of Pakistan, Ministry of Religious Affairs Vs. Dallah Real Estate and Tourism Holding Company.

Corte Suprema de Reino Unido. (2010). Dalah Real State and Tourism Holding Company Vs. Ministerio de Asuntos Religiosos, Gobierno de Pakistán.

Gómez Londoño, J. (2013). Intervención de terceros en el arbitraje nacional colombiano a la luz de las experiencias del arbitraje comercial internacional. Revista de derecho privado, 49, 1-40.

Larroumet, C. (2004). Promesse pour autrui, stipulation pour autrui et arbitrage. Revue de larbitrage, 4, 903-915.

Lee Eisen, C. (2010). What arbitration agreement? Compelling non-signatories to arbitrate. In Handbook on commercial arbitration (pp. 42-45). Huntington: Juris Publishing.

Loaiza Zapata, A. (2012). La arbitrabilidad en el nuevo estatuto de arbitraje internacional en colombia. EAFIT Journal of International Law, 3, 30-40.

Mantilla Espinosa, F. (Inédito). Aspectos relevantes de la Ley 1563 de 2012.

Mantilla Espinosa, F., Salcedo Castro, M. y Bernate Ochoa, F. (2018). La relación entre partes y terceros. En F. Mantilla Espinosa y H. Herrera Mercado (eds.), 
El arbitraje nacional frente a la corrupción. Colección Aribtraje 360. Bogotá: Documento Inédito.

Namén Vargas, W. (2000). El pacto arbitral. Revista de Derecho Privado, 5, 156-190. Park, W. (2009). Non-signatories and international contracts: An arbitrator's dilemma. In B. Macmahon (Ed.), Multiple parties in international arbitration (pp. 17-27). Oxford: Oxford University Press.

Perez, L.,E Graham, J. (2010). La teoría de los grupos de contratos aplicada a los contratos "arbitrales": una perpectiva mexicana. Revista Internacional de Arbitraje, XIII.

Perfetti Arango, D. (2010). La extensión del acuerdo arbitral a los no-signatario en el arbitraje comercial internacional. Recuperado de Escuela de arbitraje internacional http://londonoyarango.com/pdf/TesinaExtension_del_Acuerdo_Arbitral_A_No_Signatarios.pdf

Pizarro Wilson, C. (2004). Notas críticas sobre el fundamento de la fuerza obligatoria del contrato. Fuentes e interpretación del Artículo 1545 del Código Civil chileno. Revista Chilena de Derecho, 31(2), 225-237.

Pizarro Wilson, C. (2010). El efecto relativo de los contratos: partes y terceros. En C. Pizarro Wilson (ed.), Incumplimiento contractual, resolución e indemnización de daños, pp. 59-81. Bogotá: Universidad del Rosario.

Presidente de la República de Colombia. (7 de marzo de 1971). Código de Comercio colombiano. Decreto 410 de 1971. Do. 33.339

Ramírez Baquero, E. (2017). Naturaleza jurídica del pacto arbitral. En H. Herrera Mercado, y F. Mantilla Espinosa (eds.), El Proceso arbitral nacional, volumen (pp. 67-150). Bogotá: Ibáñez.

Rengifo García, E. (2012). La fiducia mercantil y pública en Colombia. Bogotá: Universidad Externado de Colombia.

Rodrígurez Azuero, S. (2013). Negocios fiduciarios. Bogotá: Legis.

Sanocki, K. (2013). Determining arbitrability of the dispute: The clear and unmistakable standard for choice of law in arbitration agreements. Journal of dispute resolution, 2013(1), 251-270.

Silva Romero, E. E Mantilla Espinosa, F. (2005). El contrato de arbitraje. Bogotá: Legis-Editorial Universidad del Rosario.

Sinisterra Páez, L. (2010). Algunos apuntes sobre la teoría del grupo de compañías como fundamento para la extensión del pacto arbitral a no signatarios. Revista de Derecho Privado, 44, 1- 32

Supreme Court United Kingdom. (3 de noviembre de 2010). Dallah Real Estate and Tourism Holding Company Vs. Government of Pakistan, Ministry of Religious Affairs. 
Tobar Ordoñez, J. (2017). Algunas consideraciones sobre la intervención de terceros en el proceso arbitral nacional. En H. Herrera Mercado y F. Mantilla Espinosa (eds.), La práctica del litigio aribtral: aspectos contractuales y procesales (pp. 315-378). Bogotá: Ibáñez.

Tribunal de Arbitramento. (4 de abril de 2000). Comercial Patrimonio S. A. contra Fiduanglo $S$. A.

Tribunal de Arbitramento. (26 de agosto de 1997). Leasing Mundial S. A. contra Fiduciaria FES S. A.

Tribunal de Arbitramento. (30 de agosto de 2012). Fideicomiso Pagos Procampo contra Productividad para el Campo S. A. - Procampo S. A. en concordato Productos Agropecuarios Ltda. y Cía. en concordato y Química Moderna S. A. - Quimor S. A. en concordato.

Vidal Olivares, A. (2010). El efecto absoluto de los contratos. En A. Vidal Olivares y C. Pizarro Wilson (eds.), Incumplimiento contractual, resolución e indemnización de daños (pp. 81-120). Bogotá: Universidad del Rosario. 
